\title{
T-wave axis deviation, metabolic syndrome and cardiovascular risk: results from the MOLI-SANI study ${ }^{\text {ts }}$
}

\author{
Deodato Assanelli, MD, ${ }^{\mathrm{a}}$ Livia Rago, MD, ${ }^{\mathrm{b}}$ Massimo Salvetti, MD, ${ }^{\mathrm{a}}$ \\ Augusto Di Castelnuovo, MSc, PhD, ${ }^{\mathrm{b}}$ Fabio Badilini, PhD, FACC, ${ }^{\mathrm{c}}$ \\ Martino Vaglio, MSc, ${ }^{\mathrm{c}}$ Francesco Zito, MD, ${ }^{\mathrm{b}}$ Maria Benedetta Donati, MD, PhD, ${ }^{\mathrm{b}}$ \\ Giovanni de Gaetano, MD, PhD, ${ }^{\mathrm{b}}$ Licia Iacoviello ${ }^{\mathrm{b}, *}$, \\ on behalf of the Moli-sani Project Investigators \\ a Department of Internal, Sport Medicine, University of Brescia, Brescia, Italy \\ b Laboratory of Genetic and Environmental Epidemiology, Fondazione di Ricerca e Cura "Giovanni Paolo II", Università Cattolica del Sacro Cuore, \\ Campobasso, Italy \\ c AMPS LLC, New York, NY, USA \\ Received 28 May 2012
}

\begin{abstract}
Early recognition of patients at increased cardiovascular risk is a major challenge. The surface electrocardiogram provides a useful platform and it has been used to propose several indexes. $\mathrm{T}$ wave axis abnormality is associated with an increased risk of cardiovascular mortality, independently of other risk factors and can be associated with the presence of the metabolic syndrome (MetS). We assessed the prevalence of $\mathrm{T}$ axis abnormalities and its relationship with MetS and its components in

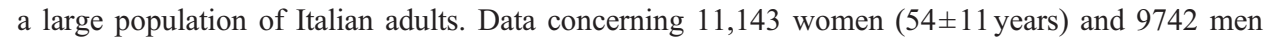
( $55 \pm 11$ years) randomly recruited from a general population (Moli-sani cohort) were analyzed. After excluding subjects with incomplete data and with history of cardiac disease or left ventricular hypertrophy, T-wave axis was normal in $74.5 \%$ of men and $80.9 \%$ of women, borderline in $23.6 \%$ and $17.3 \%$ and abnormal in $1.9 \%$ and $1.8 \%$. In subjects with MetS, the prevalence of borderline or abnormal T-wave axis deviation was higher than in subjects without MetS (in men: $26.6 \%$ vs. $22.1 \%$ and $2.5 \%$ vs. $1.7 \%$; in women: $25 \%$ vs. $15 \%$ and $2.4 \%$ vs. $1.6 \%$, respectively for borderline and abnormal levels, $\mathrm{p}<0.0001$ ). Each component of MetS increased the odds of having borderline or abnormal T-wave axis deviation by 1.21 in men and 1.31 in women. $\mathrm{T}$ wave axis deviation is associated with MetS and its individual components. These findings confirm previous reported results, expanding them to a large and representative sample of European population of Caucasian ethnicity.
\end{abstract}

(C) 2012 Elsevier Inc. All rights reserved.

Keywords: $\quad$ T-wave axes deviation; Metabolic syndrome; Estimated cardiovascular risk

\section{Introduction}

Preclinical diagnosis of cardiovascular disease has always been a difficult task; many researchers tried to find a specific marker to bridge epidemiology and clinical practice. While

\footnotetext{
is Grant Support: The MOLI-SANI Project enrolment was supported by research grants from Pfizer Foundation (Rome, Italy) and the Italian Ministry of University and Research (MIUR, Rome, Italy)-Programma Triennale di Ricerca, Decreto no. 1588.

* Corresponding author. Research Laboratories, Fondazione di Ricerca e Cura "Giovanni Paolo II", Università Cattolica del Sacro Cuore. Largo Gemelli 1, 86100 Campobasso, Italy.

E-mail address: licia.iacoviello@moli-sani.org
}

different markers have been proposed and compared, electrocardiography, together with microalbuminuria, are considered among the most cost effective. Several authors tried to introduce robust and reproducible indexes for both early diagnostic and prognostic purposes. QT/QTc duration, left ventricular hypertrophy, ventricular strain, and T-wave alternans seem to be effective markers, although a consensus on a specific marker has not been reached. Indeed, many of the proposed parameters seem effective on selected patients, but remain either difficult to measure and/or complex to compare, with a low reproducibility, and not easy to be standardised because of a high intrinsic variability. ${ }^{1,2}$ As a result of this uncertainty, qualitative parameters, still 
effective to better stratify populations, as Minnesota Code, are frequently preferred to more quantitative indexes of preclinical disease. ${ }^{3}$

T-wave axis, as derived by the 12 lead electrocardiogram, has been analysed and compared with other electrocardiographic parameters and traditional risk factors to assess as indicators of risk of cardiac events in elderly people from the Rotterdam Heart study, in a prospective study with a 4 years follow-up. ${ }^{4}$ The presence of a significant shift in the T-wave axis, derived from both the frontal and horizontal plane leads resulted as the most predictive ECG index, both for fatal and non fatal events, maintaining its predictive value even after correction for others ECG indexes. After stratification for traditional risk factors it was still indicative of fatal and non fatal cardiovascular events. It was hypothesised that this risk indicator could become a useful method both in clinical practice and in screening for patients who will develop an ischemic cardiomyopathy.

In another prospective study with a 7 years follow-up in the Cardiovascular Health Study elderly multi-ethnic population, abnormalities in T-wave axis doubled the occurrence of events and were correlated with both systolic and diastolic blood pressure, as well as with ST abnormalities and with silent myocardial infarction. ${ }^{5}$

A more recent prospective study, with a 12 years followup on a younger cohort ( 52 years) and with a low incident rate of cardiovascular events did not find a significant relation between abnormal $\mathrm{T}$-wave axis and event occurrence, in contrast with previous studies. ${ }^{6}$

A recent epidemiological report from the United States showed that abnormal T-wave axis shift is also independently associated with metabolic syndrome (MetS), suggesting the need to perform a careful electrocardiographic screening among persons with MetS for early detection of abnormal $\mathrm{T}$-wave axis in clinical practice to prevent severe and often fatal arrhythmias. ${ }^{7}$

MetS is a cluster of cardiovascular risk factors which has been affecting millions of people worldwide. In the United States approximately one-quarter of people are affected by MetS and according to the latest data on the obesity pandemic the incidence is likely to rise. ${ }^{8}$ Similar figures have been reported in Europe and in particular in Southern European countries where the prevalence of MetS is reaching worrying levels. MetS has been associated with a 2-fold increase in cardiovascular disease and a 1.5-fold increase in all-cause mortality. ${ }^{9}$

In this work we report the results of an analysis on T-wave axis deviation from the frontal plane (TDev) and its relationship with MetS and MetS components as assessed in a large community adult sample population from central Italy; as a secondary endpoint the estimated risk of CVD associated with TDev on the same sample population has been evaluated.

\section{Methods}

The cohort of the Moli-sani Study was randomly recruited between March 2005 and April 2010 in the Molise region from City Hall registries by multistage sampling across the two recruiting centres. ${ }^{10}$ A total of 24,325 subjects were enrolled by accurately trained research personnel. Admission was carefully defined and standardised and included a structured digitalized questionnaire administered to collect personal and clinical information.

The set of clinical data collected included a standard 12lead resting electrocardiogram (ECG) which was acquired with a Cardiette ${ }^{\circledR}$ ar2100-view electrocardiograph (Cardioline). Digital ECG waveforms were stored in a public domain standard (SCP format) and subsequently exported and processed by a dedicated on-screen computer application ${ }^{11}$ which embeds a proprietary measuring tool and the University of Glasgow 12-Lead ECG diagnostic algorithm. ${ }^{12}$

Metabolic syndrome (MetS) was defined based on the presence of one to five components (see Table 2 for details) according to the Adult Treatment Panel III criteria. ${ }^{13}$

Subjects were excluded in the presence of at least one of the following conditions: altered QRS duration $(n=1250)$, incomplete questionnaire $(n=235)$, history of CVD $(n=$ $1140)$, left ventricular hypertrophy $(n=1141)$, or T-axis value uncomputed because of noise $(n=195)$, or at least one MetS component missing $(n=184)$. A total of 20,885 subjects $(86 \%), 11,143$ women (mean age $55 \pm 11$ ) and 9742 men (mean age $55 \pm 11$ ) were analyzed. The value of $\mathrm{T}$-wave axis in the frontal plane was categorized in three groups as follows ${ }^{2}$ :

- Normal: $15^{\circ} \leq \mathrm{TDev} \leq 75^{\circ}$

- Borderline: $75^{\circ}<\mathrm{TDev} \leq 105^{\circ}$ or $-15^{\circ} \leq \mathrm{TDev}<15^{\circ}$

- Abnormal: $-180^{\circ} \leq \mathrm{TDev}<-15^{\circ}$ or $105^{\circ}<\mathrm{TDev} \leq 180^{\circ}$

Generalized linear models were used for testing the association of the TDev classification with general characteristics of MetS. Multivariable logistic regression analysis was applied to quantify the association of MetS or components of MetS with status of borderline or abnormal TDev in comparison with normal conditions. Estimates of global individual CVD risk were calculated applying the equations defined in the CUORE project. ${ }^{14,15}$

\section{Results}

General characteristics of men and women included in the study are illustrated in Table 1 whereas Table 2 summarizes the results categorized by MetS components. MetS was prevalent in $27 \%(n=2643)$ of men and in $24 \%$ $(n=2663)$ of women. TDev was categorized as normal in $74.5 \%$ of men and in $80.9 \%$ of women, borderline in $23.6 \%$ and $17.3 \%$ and abnormal in $1.9 \%$ and $1.8 \%$. In men, the prevalence of borderline and abnormal TDev was higher in the presence of MetS (26.6\% vs. $22.1 \%$ and $2.5 \%$ vs. $1.7 \%$, respectively for borderline and abnormal TDev; $\mathrm{p}<0.0001$, Table 1a). The prevalence of normal TDev decreased from $84 \%$ to $66 \%$ when the number of MetS components increased from zero to five (Table $2 \mathrm{a}$ ), and each additional MetS component increased the odds of having borderline or abnormal TDev by 1.21. Similarly, in women, the 
Table 1

Males and Females characteristics by MetS status.

\begin{tabular}{|c|c|c|c|c|}
\hline \multirow{2}{*}{$\frac{\mathrm{MEN}}{\text { Characteristics }}$} & \multicolumn{4}{|l|}{ Metabolic Syndrome } \\
\hline & All 9742 & Absent $7099(73 \%)$ & Present $2643(27 \%)$ & p-value Age adjusted \\
\hline \multicolumn{5}{|l|}{$a$} \\
\hline Age, years (sd) & $55(11)$ & $54(12)$ & $57(11)$ & $<.0001 \#$ \\
\hline BMI, kg/m² (sd) & $28.2(4.1)$ & $27.1(3.4)$ & $31.1(4.3)$ & $<.0001$ \\
\hline Heart rate $\mathrm{b} / \mathrm{m}$ & $66(10)$ & $65(10)$ & $68(11)$ & $<.0001$ \\
\hline QRS complex duration, ms & $91.1(8)$ & $91.0(8)$ & $91.2(8)$ & 0.21 \\
\hline T-wave axis deviation $n,(\%)$ & & & & $<.0001$ \\
\hline Normal & $7256(74.5)$ & $5408(76.2)$ & $1848(69.9)$ & \\
\hline Borderline & $2301(23.6)$ & $1572(22.1)$ & $729(27.6)$ & \\
\hline Abnormal & $185(1.9)$ & $119(1.7)$ & $66(2.5)$ & \\
\hline WOMEN & \multicolumn{4}{|l|}{ Metabolic syndrome } \\
\hline Characteristics & All 11,143 (100\%) & Absent $8480(76 \%)$ & Present $2663(24 \%)$ & p-value Age adjusted \\
\hline \multicolumn{5}{|l|}{$b$} \\
\hline Age, years (sd) & $54(11)$ & $53(11)$ & $60(11)$ & $<.0001 \#$ \\
\hline BMI, kg/m² (sd) & $27.7(5.3)$ & $26.5(4.7)$ & $31.7(5.1)$ & $<.0001$ \\
\hline Heart rate $\mathrm{b} / \mathrm{m}$ & $69(10)$ & $68(10)$ & $70(11)$ & $<.0001$ \\
\hline QRS complex duration, ms & $86.1(8)$ & $86.0(8)$ & $86.4(7)$ & 0.014 \\
\hline T-wave axis deviation $\mathrm{n}, \%$ & & & & $<.0001$ \\
\hline Normal & $9012(80.9)$ & $7078(83.5)$ & $1934(72.6)$ & \\
\hline Borderline & $1929(17.3)$ & $1264(14.9)$ & $665(25.0)$ & \\
\hline Abnormal & $202(1.8)$ & $138(1.6)$ & $64(2.4)$ & \\
\hline
\end{tabular}

prevalence of borderline or abnormal TDev was higher in the presence of MetS $(25 \%$ vs. $15 \%$ and $2.4 \%$ vs. $1.6 \%$, respectively for borderline and abnormal TDev; $p<0.0001$, Table 1b). The prevalence of normal TDev decreased from
$89 \%$ to $74 \%$ when the number of MetS components increased from zero to five (Table 2b), and each additional MetS component increased the odds of having borderline or abnormal TDev by 1.31 .

Table 2

Metabolic syndrome components according to T-wave axis deviation in women.

\begin{tabular}{|c|c|c|c|c|}
\hline \multirow{2}{*}{$\begin{array}{l}\text { MEN } \\
\text { Characteristics }\end{array}$} & \multicolumn{4}{|l|}{$\mathrm{T}$ - wave axis deviation } \\
\hline & Normal n=7256 (74\%) & Borderline $\mathrm{n}=2301(24 \%)$ & Abnormal $n=185(2 \%)$ & p-value Age adjusted \\
\hline \multicolumn{5}{|l|}{$a$} \\
\hline Waist circumference $\geq 102 \mathrm{~cm}$ & $1795(67 \%)$ & $826(31 \%)$ & $69(3 \%)$ & $<.0001$ \\
\hline Triglycerides $\geq 150 \mathrm{mg} / \mathrm{dl}$ & $2570(73 \%)$ & $864(25 \%)$ & $63(2 \%)$ & 0.11 \\
\hline $\mathrm{HDL}<40 \mathrm{mg} / \mathrm{dl}$ & $1064(72 \%)$ & $373(25 \%)$ & $29(2 \%)$ & 0.074 \\
\hline $\mathrm{SBP} \geq 130$ or $\mathrm{DBP} \geq 85 \mathrm{~mm} \mathrm{Hg}$ & $5545(73 \%)$ & $1893(25 \%)$ & $166(2 \%)$ & $<.0001$ \\
\hline Fasting glucose $\geq 110 \mathrm{mg} / \mathrm{dl}$ & $1905(71 \%)$ & $697(26 \%)$ & $72(3 \%)$ & $<.0001$ \\
\hline Number of MetS components & & & & $<.0001$ \\
\hline 0 & $938(84 \%)$ & $175(16 \%)$ & $6(0.5 \%)$ & \\
\hline 1 & $2335(76 \%)$ & $629(21 \%)$ & $50(2 \%)$ & \\
\hline 2 & $2135(72 \%)$ & $768(26 \%)$ & $63(2 \%)$ & \\
\hline 3 & $1227(70 \%)$ & $481(27 \%)$ & $44(3 \%)$ & \\
\hline 4 & $512(71 \%)$ & $195(27 \%)$ & $19(3 \%)$ & \\
\hline 5 & $109(66 \%)$ & $53(32 \%)$ & $3(2 \%)$ & \\
\hline WOMEN & \multicolumn{4}{|l|}{ T-wave axis deviation } \\
\hline Characteristics & Normal n=9012 (81\%) & Borderline $\mathrm{n}=1929(17 \%)$ & Abnormal $n=202(2 \%)$ & P-value Age adjusted \\
\hline \multicolumn{5}{|l|}{$b$} \\
\hline Waist circumference $\geq 88 \mathrm{~cm}$ & $4894(76 \%)$ & $1392(22 \%)$ & $146(2 \%)$ & $<.0001$ \\
\hline Triglycerides $\geq 150 \mathrm{mg} / \mathrm{dl}$ & $1528(75 \%)$ & $474(23 \%)$ & $40(2 \%)$ & $<.0001$ \\
\hline $\mathrm{HDL}<50 \mathrm{mg} / \mathrm{dl}$ & $1507(76 \%)$ & $435(22 \%)$ & $35(2 \%)$ & $<.0001$ \\
\hline $\mathrm{SBP} \geq 130$ or $\mathrm{DBP} \geq 85 \mathrm{~mm} \mathrm{Hg}$ & $5447(78 \%)$ & $1409(20 \%)$ & $159(2 \%)$ & $<.0001$ \\
\hline Fasting glucose $\geq 110 \mathrm{mg} / \mathrm{dl}$ & $1111(76 \%)$ & $321(22 \%)$ & $39(3 \%)$ & $<.0001$ \\
\hline Number of MetS components & & & & $<.0001$ \\
\hline 0 & $1884(89 \%)$ & $201(10 \%)$ & $21(1 \%)$ & \\
\hline 1 & $2549(86 \%)$ & $388(13 \%)$ & $38(1 \%)$ & \\
\hline 2 & $2645(78 \%)$ & $675(20 \%)$ & $79(2 \%)$ & \\
\hline 3 & $1242(73 \%)$ & $418(25 \%)$ & $37(2 \%)$ & \\
\hline 4 & $538(71 \%)$ & $196(26 \%)$ & $23(3 \%)$ & \\
\hline 5 & $154(74 \%)$ & $51(24 \%)$ & $4(2 \%)$ & \\
\hline
\end{tabular}


After adjustment for age, there was a significant association between TDev and cardiovascular risk score at 10 years, in both genders. In men, after further adjustment for cigarette smoking, social status, physical activity and QRS duration, TDev was associated with an increase in the risk to develop a cardiovascular event in 10 years of 1.38 and 4.44 , respectively for borderline and abnormal TDev. In women, the association reached significant levels only in univariate analysis (estimated risk at 10 years 1.37 and 1.78 , respectively for borderline and abnormal TDev).

\section{Discussion}

In this work, the epidemiological usefulness of T-wave axis in the frontal plane in a large Italian Mediterranean cohort has been analyzed with the primary goal to critically compare the incidence of deviations of this parameter with that observed on studies on other populations, and with special emphasis on metabolic syndrome. The potential incremental value of TDev as an alternative risk factor of cardiovascular events has also been assessed.

Our findings confirm what was previously reported, i.e. they demonstrate a significant association between an abnormal TDev with MetS and its single components. ${ }^{5}$ In addition, our analysis showed that an abnormal TDev is strongly related to the estimated cardiovascular risk at 10 years, as calculated with the CUORE algorithm. ${ }^{14,15}$

Table 3 summarized the results from five studies (including our report): the four manuscripts in which TDev was quantified concur on the percentage of apparently healthy subjects with abnormal TDev which increases from about to $2 \%-3 \%$ in middle-aged adults (about 50 years) to $10 \%$ in elderly (age about 70 years). Of note, one of the studies referenced in the Table is a recent research on the Nhanes cohort of a relatively young and bi-ethnic population, which demonstrated a graded relation between T-wave abnormalities on the frontal axis and metabolic syndrome occurrence, as well as with individual components of the metabolic syndrome. ${ }^{7}$ This result seems to indicate that in a percentage of patients with MetS an abnormality on the surface ECG could predict preclinical ischemia or myocardial remodeling predisposing to potentially dangerous arrhythmias. Our results, obtained in a larger cohort of Caucasians from the Mediterranean region are in agreement with results obtained in the NHANES study on an American population, with respect to both the reported incidence of abnormal TDev and the significant linearly-related increase of TDev abnormalities associated with the presence of one or more component of MetS. The total incidence of abnormal TDev associated with MetS observed in the NHANES cohort was about $5 \%{ }^{7}$ which is about double than that observed in our study (Table 1); this difference could be related to the genetically different population but also to the higher incidence of cardiovascular disease in North America as compared to the Mediterranean region.

In our population, the baseline percentage of abnormal TDev was found to be around $2 \%$, which is close to the value $(3 \%)$ observed on the NHANES cohort. $^{7}$ This finding is consistent with the fact that both the Moli-sani and the Nhanes are consistently younger populations than that described in the Rotterdam Heart Study where the incidence of abnormal TDev reached $10 \% .{ }^{4}$ Some of the differences between these findings are probably related to ethnicity, although the prevalence of an abnormal TDev directly related to race had not yet been demonstrated. Variants related to the methodology to determine T-wave axis, and the interaction between this parameter and other ECG measurements could also explain different results in the literature.

T-wave axis deviation reflects abnormal ventricular repolarization which is indicative of subclinical myocardial pathology. This parameter has been associated with an increased risk of coronary heart disease (CHD) and total mortality, independent of other cardiovascular factors and particularly in elderly populations. ${ }^{4}$

In four of the studies reported in Table 3, TDev resulted in an effective risk indicator, probably a marker of preclinical heart disease, whereas in one it did not provide a better stratification as compared to traditional risk factors. ${ }^{6}$ Results from Rautaharju et al. suggest that TDev is a marker for subclinical disease and an independent indicator for the risk of incident CHD events in older men and women. ${ }^{5}$

The different occurrence of fatal and non fatal events is obviously related to the age of the population but it could also be explained by different genetic substrate, environmental pattern and by the selection of the cohort (with or without exclusion of patients affected by coronary artery disease). What seems clear when comparing different studies is that the incidence of abnormal TDev increases when the mean age of the cohort evaluated is higher (Table 3), and that in both American and European cohorts an abnormal TDev is related with the metabolic syndrome and with each of its component. ${ }^{7,9}$ This finding seems to favor a pathogenetic interpretation of the mechanism determining the fatal and non fatal occurrence of events over time in patients with abnormal TDev at enrollment.

If confirmed, it would be of great interest to know whether an altered TDev in patients with MetS can be a more

Study cross-comparison on TDev and cardiovascular risk associated with TDev.

\begin{tabular}{|c|c|c|c|c|c|}
\hline & \# subjects & Mean age (years) & Race & \%abn. TDev & Cardiovascular risk \\
\hline Kors & 5781 & 69 & Caucasian & 10.5 & 4.04 \\
\hline Vaidean & 12,256 & 54 & Caucasian and Afro-American & uncomputed & non significant \\
\hline Rautahariu & 4173 & 72 & multi-ethnic & 12 & 2.88 \\
\hline Faramawi & 3810 & 59 & Caucasian, black, others & 2.97 & not inspected \\
\hline Assanelli & 24,325 & 55 & Caucasian & $1.8-2.8$ & $>1.5$ \\
\hline
\end{tabular}


critical indicator of heart disease, than the presence of MetS with normal TDev. The knowledge of a mechanism in patients with MetS and TDev abnormalities could provide better understand whether an ischemic or arrhythmic substrate could be the trigger of events in both conditions.

Patients with an abnormal $\mathrm{T}$ wave axis should be evaluated with Holter monitoring to detect arrhythmias, with ECG stress-test to detect silent ischemic events and with echocardiogram to exclude ventricular remodeling. This in-depth screening should help to understand the cause of T-wave deviation not only on an epidemiological perspective, but with a clinical individually tailored evaluation. The better knowledge of the causes of T-wave axis abnormalities could allow physicians to effectively treat individual patients to avoid or delay the occurrence of major cardiovascular events.

\section{Acknowledgments}

The Authors thank Associazione Cuore-Sano (Campobasso, Italy), IL Instrumentation-Laboratory (Milano, Italy), Derby-Blue (San Lazzaro di Savena, Bologna, Italy), Caffè Monforte (Campobasso, Italy) and Sepinia SpA (Sepino, Italy) for their support to the MOLI-SANI project.

\section{References}

1. Savelieva I, Yi G, Guo X, Hnatkova K, Malik M. Agreement and reproducibility of automatic versus manual measurement of QT interval and QT dispersion. Am J Cardiol 1998;81:471.

2. Funck-Brentano C, Jaillon P. Rate-corrected QT interval: techniques and limitations. Am J Cardiol 1993;72:B17.
3. Auer R, Bauer DC, Marques-Vidal P, et al. Association of major and minor ECG abnormalities with coronary heart disease events. JAMA 2012;307:1497.

4. Kors JA, de Bruyne MC, Hoes AW, et al. T-axis as an indicator of risk of cardiac events in elderly people. Lancet 1998;352:601.

5. Rautaharju PM, Nelson JC, Kronmal RA, et al. Usefulness of T-axis deviation as an independent risk indicator for incident cardiac events in older men and women free from coronary heart disease (the Cardiovascular Health Study). Am J Cardiol 2001;88:118.

6. Vaidean GD, Rautaharju PM, Prineas RJ, et al. The association of spatial $\mathrm{T}$ wave axis deviation with incident coronary events. The ARIC cohort. BMC Cardiovasc Disord 2005;5:1.

7. Faramawi MF, Sall M, Abdul Kareem MY. The association of the metabolic syndrome with T-wave axis deviation in NHANES III. Ann Epidemiol 2008;18:702.

8. Ford ES, Giles WH, Dietz WH. Prevalence of the metabolic syndrome among US adults: findings from the third National Health and Nutrition Examination Survey. JAMA 2002;287:356.

9. Mottillo S, Filion KB, Genest J, et al. The metabolic syndrome and cardiovascular risk a systematic review and meta- analysis. J Am Coll Cardiol 2010;56:1113.

10. Iacoviello L, Bonanni A, Costanzo S, et al. The Moli-sani Project, a randomized, prospective cohort study in the Molise region in Italy; design, rationale and objectives. Italian J. Public Health 2007;4:110.

11. Badilini F, Sarapa N. Implications of methodological differences in digital electrocardiogram interval measurement. J Electrocardiol 2006; 39:S152.

12. Macfarlane PW, Devine B, Latif S, McLaughlin S, Shoat DB, Watts MP. Methodology of ECG interpretation in the Glasgow program. Methods Inf Med 1990;29:354.

13. Executive summary of the third report of the National Cholesterol Education Program (NCEP) Expert Panel on Detection, Evaluation, and Treatment of High Blood Cholesterol in Adults (Adult Treatment Panel III). JAMA 2001;285:2486.

14. http://www.cuore.iss.it/cuore_exe/cuore_exe.asp.

15. Ferrario M, Chiodini P, Chambless LE, et al. Prediction of coronary events in a low incidence population. Assessing accuracy of the CUORE Cohort Study prediction equation. Int J Epidemiol 2005;34:413. 